\title{
Identifying variable $\gamma$-ray sources through radio observations
}

\author{
J.M. Paredes (jmparedes@ub.edu) \\ Universitat de Barcelona \\ J. Martí \\ Universidad de Jaén \\ D.F. Torres \\ Lawrence Livermore National Laboratory
}

G.E. Romero

Instituto Argentino de Radioastronomía

FCAGLP, Universidad Nacional de la Plata

\author{
J.A. Combi \\ Universidad de Jaén
}

V. Bosch-Ramon and J. García-Sánchez

Universitat de Barcelona

\begin{abstract}
We present preliminary results of a campaign undertaken with different radio interferometers to observe a sample of the most variable unidentified EGRET sources. We expect to detect which of the possible counterparts of the $\gamma$-ray sources (any of the radio emitters in the field) varies in time with similar timescales as the $\gamma$-ray variation. If the $\gamma$-rays are produced in a jet-like source, as we have modelled theoretically, synchrotron emission is also expected at radio wavelengths. Such radio emission should appear variable in time and correlated with the $\gamma$-ray variability.
\end{abstract}

Keywords: $\gamma$-ray sources, radio sources, microquasars, microblazars.

\section{Introduction}

The Third EGRET Catalog (Hartman et al. 1999) lists 271 point sources. About two thirds of them have no conclusive counterparts at lower frequencies. Even worse, 40 of them do not show any positional coincidence (within the $95 \%$ EGRET contour) with possible $\gamma$-ray emitting objects known in our galaxy (Romero, Benaglia, \& Torres 1999, Torres et al. 2001). In order to understand the origin of all these unidentified detections, their variability status is of fundamental importance. Classic known models for $\gamma$-ray sources in our galaxy would produce non-variable sources during the timescale of observations. This is the case of pulsars (Thompson 2001), supernova remnants in interaction with molecular clouds (Torres et al. 2003) and microquasar jets in interaction with high density interstellar medium (Bosch-Ramon et al. 2004a). Alternatively, if some of the sources are produced by compact

(c) 2017 Kluwer Academic Publishers. Printed in the Netherlands. 
objects, such as isolated magnetized black holes (Punsly et al. 2000) or microquasars (Paredes et al. 2000), high levels of flux variability can be expected.

According to recent theoretical models, the observed variable $\gamma$ ray emission could be associated with galactic compact objects such as X-ray binary systems and isolated black holes. A model considers the so-called microquasar and microblazar X-ray binaries, in which the optical/UV photons from an optical star are inverse Compton up-scattered by relativistic electrons in the jets emanating from the accretion disc of a black hole/neutron star companion. Variability is then naturally induced by the precesion of the jets, eccentricity of the orbit and/or variable accretion rates. Moreover, it should be also detectable at radio wavelengths where synchrotron radio photons are produced by the same relativistic electrons. A semi-analytical model (see Fig. 1) of this and other processes, in a microquasar scenario, is currently being developed by us with the purpose of understanding the spectral energy distribution of EGRET sources across the whole electromagnetic spectrum (Bosch-Ramon et al. 2004b). The non-thermal spectral energy distribution has two peaks, one from the synchrotron jet emission, at radio-IR energies, and other from Comptonization of seed photons (stellar, coronal, disc and/or synchrotron photons), at MeV$\mathrm{GeV}$ energies. The amplitude of the radio variation would be somewhere between 10\%, as observed in the microquasar LS5039/3EG J1824-1514 (Ribó 2002, Ribó et al. 2004), and one order of magnitude, as predicted in some microblazar models (Romero et al. 2002, Kaufman-Bernadó et al. 2002). The time scales could range between the orbital and the precession periods typical of X-ray binaries (from days to a few months).

Another possibility for a variable galactic $\gamma$-ray source is that of an isolated black hole. It has been suggested that such objects could radiate $\gamma$-rays due to spherical accretion from the interstellar medium (Dermer 1997) or thanks to a charged magnetosphere (Punsly 1998, Punsly et al. 2000). Finally, yet another possibility is the $\gamma$-ray emission produced in the disc of a binary system formed by a pulsar and a massive star. The pulsar accelerates charged particles in its magnetosphere, which ultimately collide against the accretion disc formed by the matter from the companion star. This collision yields hadronic (pp) interactions, and variability naturally results as a consequence of the formation and loss of the accretion disc along the binary orbit. A situation like this was explored in the case of A0535+26 by Romero et al. (2001), and was found able to explain the observations for $3 \mathrm{EG} 0542+2610$. In this case, however, the $\gamma$-ray emission is mainly hadronic, and we do not expect intense radio counterparts. All the 


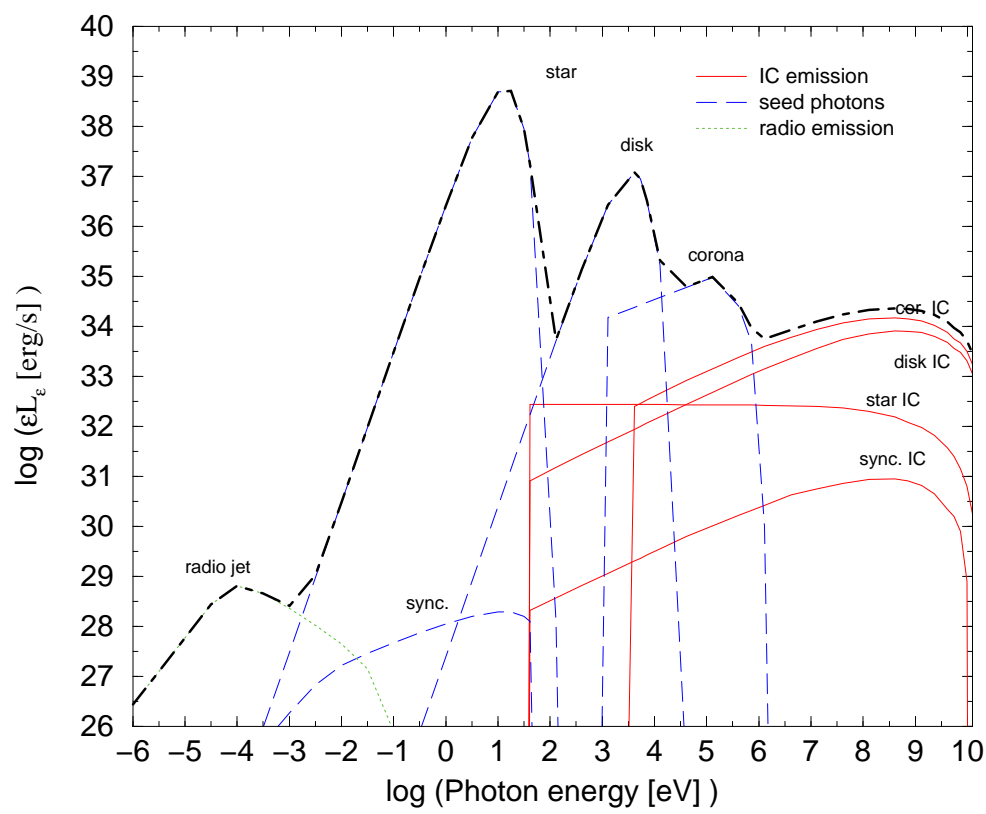

Figure 1. Spectral energy distribution of a typical microquasar emitting up to EGRET energies (Bosch-Ramon et al. 2004b).

previous models could produce variability indices in agreement with those determined by Torres et al. (2001).

Identifying the most variable sources -and particularly those which simultaneously show steep spectral indices at $\gamma$-rays - is bound to yield very interesting discoveries. In this work we report preliminary results of a search for variable radio counterparts of $\gamma$-ray variable EGRET sources using different radio interferometers.

\section{Selected sources to be studied}

From the original set of unidentified EGRET sources at $|b| \leq 10^{\circ}$, a large fraction $\sim 50 \%$ (40 sources) remains without any known possible galactic counterpart (Romero, Benaglia, \& Torres 1999). Since it is most unlikely that all these sources are extragalactic, they must encompass a population of variable galactic $\gamma$-ray sources yet to be discovered.

To quantify the variability status of these sources we use the $I$ index, which establishes how variable a source is with respect to the pulsar population. The reader is referred to Torres et al. (2001), and Torres, Pessah, \& Romero (2001) for details and comparisons with other variability estimators proposed in the literature. Sources with $I>2.5$ 
Table I. Unidentified 3EG sources without known possible galactic counterparts and visible from WSRT.

\begin{tabular}{lcccccc}
\hline $\begin{array}{l}\gamma \text {-Source } \\
(3 \mathrm{EG} \mathrm{J})\end{array}$ & $\begin{array}{c}l \\
(\mathrm{deg})\end{array}$ & $\begin{array}{c}b \\
(\mathrm{deg})\end{array}$ & $\Delta \theta$ & $\Gamma$ & $\begin{array}{c}\left\langle F_{\gamma}\right\rangle \times 10^{-8} \\
\left(\mathrm{ph} \mathrm{cm}^{-2} \mathrm{~s}^{-1}\right)\end{array}$ & $I$ \\
\hline $1735-1500$ & 10.73 & 9.22 & 0.77 & $3.24 \pm 0.47$ & 19.0 & 8.86 \\
$1746-1001$ & 16.34 & 9.64 & 0.76 & $2.55 \pm 0.18$ & 29.7 & 3.19 \\
$1810-1032$ & 18.81 & 4.23 & 0.39 & $2.29 \pm 0.16$ & 31.5 & 2.61 \\
$1812-1316$ & 16.70 & 2.39 & 0.39 & $2.29 \pm 0.11$ & 43.0 & 2.60 \\
$1828+0142$ & 31.90 & 5.78 & 0.55 & $2.76 \pm 0.39$ & 30.8 & 5.33 \\
$1834-2803$ & 5.92 & -8.97 & 0.52 & $2.62 \pm 0.20$ & 17.9 & 2.83 \\
$1904-1124$ & 24.22 & -8.12 & 0.50 & $2.60 \pm 0.21$ & 22.5 & 2.91 \\
$1928+1733$ & 52.91 & 0.07 & 0.75 & $2.23 \pm 0.32$ & 38.6 & 3.99 \\
$2035+4441$ & 83.17 & 2.50 & 0.54 & $2.08 \pm 0.26$ & 39.1 & 3.35 \\
\hline
\end{tabular}

are already $3 \sigma$ away from the statistical variability of pulsars, and we can be confident in that its variability status might be indicative of its ultimate nature. Selected EGRET sources that, being not in positional coincidence with any known plausible galactic counterpart, are most likely variable, are listed in Table I. There, we give their 3EG name, their galactic coordinates, the error in their position, in degrees (assumed to be the $95 \%$ confidence contours given by the 3EG catalog), their gamma spectral index, $\Gamma$ (such that the photon distribution is given by $N(E) \propto E^{-\Gamma}$ ), their EGRET flux and the index of variability $I$. In a first step, we included only those with smaller EGRET position error and/or without hour angle limitation for the interferometer to be used. Their range of variability index is 2.61 to 5.33 which can be considered as very significant. The typical size of the EGRET 95\% confidence contours is $\sim 0.6^{\circ}$. It is also worth noticing that the mean value of the EGRET spectral indices is quite steep, steeper than the steepest pulsar spectrum known.

The sample in Table I was initially investigated for possible radio counterparts (although no information on source variability could be gathered at this stage). We constructed radio maps at the $20 \mathrm{~cm}$ wavelength using data from the NRAO VLA Sky Survey ${ }^{1}$ (NVSS, Condon et al. 1998) for each of these EGRET detections and classified the radio sources within each EGRET confidence contours. There are between 7

\footnotetext{
1 The National Radio Astronomy Observatory is a facility of the National Science Foundation operated under cooperative agreement by Associated Universities, Inc.
} 
and 30 possible radio counterparts (mostly uncatalogued) in each case that need to be monitored in order to establish which of them -if anyis varying in time.

The $\gamma$-ray variability of all our targets clearly points out to a compact object as the origin of the high energy emission. 3EG J1828+0142 has a previously known possible extragalactic counterpart although outside the 95\% EGRET confidence contour. It is an active galactic nucleus referred to as J1826+0149 by Sowards-Emmerd et al. (2003), who report it to be a flat spectrum radio quasar. Halpern et al. (2003) also found this association plausible. However, an alternative galactic model also exists for this source (Punsly et al. 2000, Butt et al. 2002, Combi et al. 2001, Bosch-Ramon et al. 2004c). In another case, 3EG J1735-1500, a radiogalaxy within the location error box has been proposed as a counterpart although alternative candidates exist as well (Combi et al. 2003, Bosch-Ramon et al. 2004c).

\section{Radio Observations}

In order to identify the origin of our selected $\gamma$-ray sources, we have undertaken a programme of radio observations using different interferometers. Most of our data has been obtained with the Westerbork Synthesis Radio Telescope (WSRT) at the $21 \mathrm{~cm}$ wavelength. In addition, the Very Large Array (VLA) of the NRAO was also used for an initial test of our identification strategy. With this approach, we expect to detect which of the possible counterparts of the $\gamma$-ray sources (any of the radio emitters in the field) varies in time with similar timescales to that of the $\gamma$-ray variation. If the $\gamma$-ray emission is produced in a jet-like source, for instance, synchrotron emission leading to radio detections, will also show a time dependence. Flux densities of at least $\sim 1 \mathrm{mJy}$ are expected by analogy with radio emitting X-ray binaries. The current status of our identification project is described in the following sections.

\subsection{WSRT MULTI-EPOCH OBSERVATIONS}

The WSRT observations were conducted so far for two of the targets in Table I, namely 3EG J1928+1733 and 3EG J2035+441. We observed them on 2003, June 15 (WSRT1) and September 20 (WSRT2), thus with a time separation of three months. In both cases we observed at the $21 \mathrm{~cm}$ wavelength, with a $20 \mathrm{MHz}$ bandwitdth, a correlator setup of 64 channels, 4 polarizations and 8 bands. The array of antennas was always in the same configuration in order to facilitate variability

studies, with $72 \mathrm{~m}$ of separation between the dishes of the two movable 
pairs of antennae. Calibrator sources were observed one hour before and after each observing run following the standard procedures at the WSRT.

The first observing epoch was devoted to obtain a $21 \mathrm{~cm}$ mosaicked full track of $12 \mathrm{~h}$ duration for each EGRET field with the idea of using it as a template for future variability studies. The different pointings, seven per EGRET field, were arranged with a hexagonal packing covering a field of $\sim 2^{\circ}$ squared. In most cases, this should be enough to fully cover the $95 \%$ confidence contour of the EGRET position. The pointings were corrected for the primary beam response before being combined into a mosaic. This process increases significantly the noise in the map far from the central pointing positions. The sky positions covered by more than one pointing were weighted with the inverse square of the rms noise before averaging and combining them. The resulting mosaic has a typical $\mathrm{rms}$ noise of $\sim 0.2 \mathrm{mJy}^{\text {beam }}{ }^{-1}$ over most of its solid angle. A circular restoring beam of $45^{\prime \prime}$ was used in order to make easier the comparison with NVSS images.

During the second observing epoch, the two EGRET fields were mosaicked during a single $12 \mathrm{~h}$ track with the same instrumental setup of the first epoch. Sensitivity with the mosaicked snapshots was, of course, lower but well enough to control the variability of already known NVSS sources as well as to look for new fainter radio variables in the field (up to $\sim 1 \mathrm{mJy}$ level). Subtraction maps between the two epochs, in the image plane, were found to be a suitable tool to look for possible variables in the field.

\subsection{VLA OBSERVATIONS}

A Director Discretionary Time (DDT) observation was also conducted at the VLA as a test of our identification strategy for this project. The target was 3EG J1812-1316, whose field we mosaicked with four pointings only during a single observing epoch (2003 February 10). The VLA data were taken at the $20 \mathrm{~cm}$ wavelength in D configuration with the idea of comparing them directly with the NVSS images. The

corresponding results are discussed below together with those from the WSRT.

\section{Results}

Examples of our WSRT mosaics are presented in Figs. 2 and 3. Both correspond to our first observing epoch and they have been computed using uniform weight. Many radio sources are detected in each WSRT mosaic $(\sim 100)$ up to a brightness limit of $0.5 \mathrm{mJy}$. 
The comparison of our two WSRT epochs revealed initially no strongly flaring sources, at least in time scales of months. In Tables II and III, we list the WSRT positions and flux densities of the possible radio variables detected (six in each field) as measured with the IMFIT task of the AIPS package. Some of the variable candidates reported here, with $\leq 10 \%$ amplitude, are barely above the uncertainty in absolute flux calibration and further observations are required to confirm them. In spite of this, the comparison of our WSRT maps with the NVSS has actually revealed remarkable cases of apparent radio transient, or flaring. At least one radio transient source, in time scales of years, is present among our variable candidates in both EGRET fields. These interesting objects are marked with an asterisk in Tables II and III, where the corresponding NVSS flux density at the $20 \mathrm{~cm}$ wavelength is also included (upper limits are $3 \sigma$ ). In addition, we illustrate them as well in Figs. 4 and 5. The small wavelength difference, between the WSRT and the NVSS, is not likely to affect the reality of the proposed radio transient sources for physically plausible spectral indices.

The comparison with the NVSS has been also very useful to identify radio variable candidates in our single epoch VLA observations of 3EG J1812-1316, again in time scales of years. The results are presented in Table IV together with the NVSS data. Two of the sources in the field, also marked with an asterisk, appear as reliable variable radio sources. It is important to mention here that our VLA data was obtained in the same D configuration of the array as the NVSS, hence comparison is straightforward.

Multiwavelength follow-up observations (X-ray, infrared, optical, and eventually VLBI) of these so-detected confident candidates can ultimately yield to the discovery of new microquasars or other $\gamma$-rayemitting galactic compact objects. We have initially inspected both the Digitized Sky Survey (DSS) and other databases (e.g. 2MASS) looking for possible counterparts of our discovered radio variables. The strong absorption in the Galactic plane at optical wavelengths makes the search for an optical counterpart very difficult. In the infrared, the 2MASS has evidence of a few sources having $K \sim 15$ that could be consistent with some of our \pm 1 " radio positions. However, a confirmation with deep $K$-band images is strongly necessary specially in cases where the 2MASS revealed no clear infrared source and a proposal for this goal has been recently submitted to the Calar Alto $3.5 \mathrm{~m}$ telescope in Spain. To determine the nature of the candidates, either galactic or extragalactic, infrared spectroscopy studies on the near infrared counterparts will be performed. Similarly, the most interesting radio variable candidates have been proposed for Chandra observations in the X-ray band. 


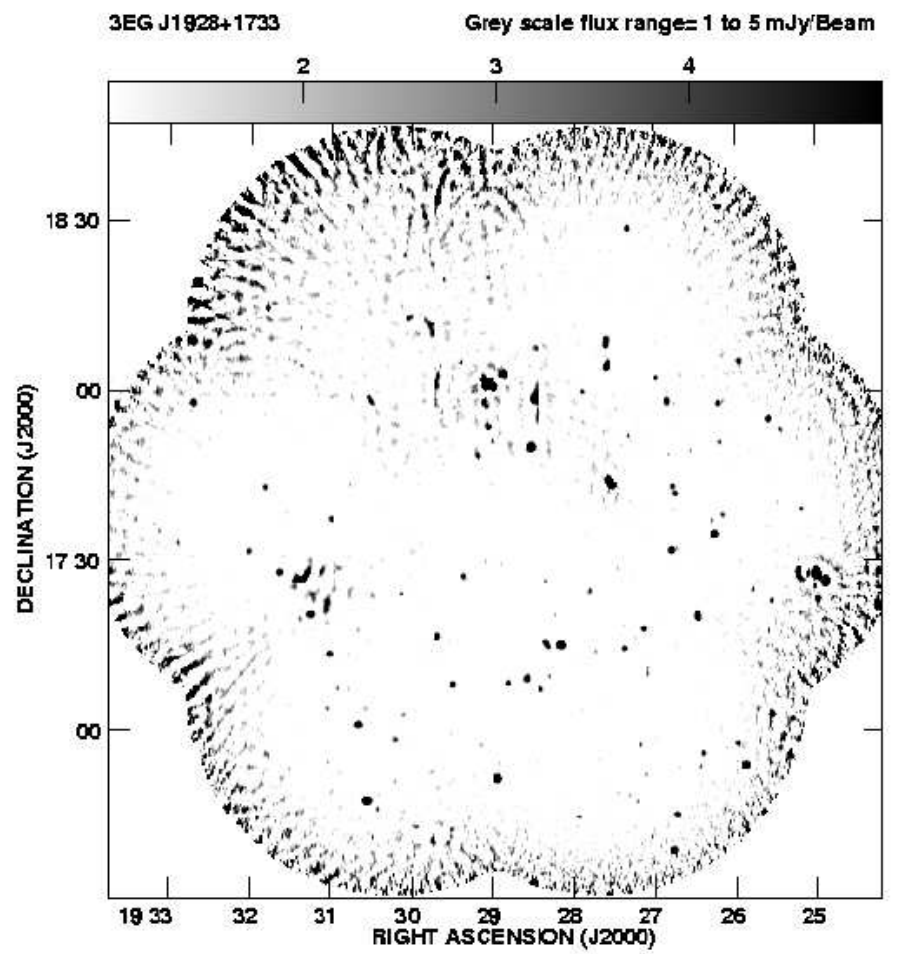

Figure 2. WSRT mosaic of the 3EG J1928+1733 field at $21 \mathrm{~cm}$ taken on 2003 June 15 , the first of our two WSRT observing epochs. A circular restoring beam of 45 " has been used for easy comparison with the NVSS.

Table II. Possible variable radio sources in the 3EG J1928+1733 field. The most reliable variables are marked with $\left({ }^{*}\right)$.

\begin{tabular}{ccccc}
\hline $\begin{array}{c}R A(2000) \\
(\mathrm{h} \mathrm{m} \mathrm{s})\end{array}$ & $\begin{array}{c}D E C(2000) \\
\left({ }^{\prime}, "\right)\end{array}$ & $\begin{array}{c}\text { NVSS } \\
(\mathrm{mJy})\end{array}$ & $\begin{array}{c}\text { WSRT1 } \\
(\mathrm{mJy})\end{array}$ & $\begin{array}{c}\text { WSRT2 } \\
(\mathrm{mJy})\end{array}$ \\
\hline $192735.56 \pm 0.03$ & $+180438.2 \pm 0.6$ & $38.1 \pm 2.1$ & $30.8 \pm 0.8$ & $25.6 \pm 1.1$ \\
$192809.95 \pm 0.01$ & $+171517.4 \pm 0.1$ & $55.0 \pm 1.7$ & $62.8 \pm 0.6$ & $61.0 \pm 0.8$ \\
$193031.40 \pm 0.07^{a}$ & $+175814.4 \pm 0.7$ & $5.2 \pm 0.6$ & $14.6 \pm 0.6$ & $22.8 \pm 1.0$ \\
$193100.91 \pm 0.03^{*}$ & $+171330.7 \pm 0.5$ & $2.5 \pm 0.5$ & $13.3 \pm 0.5$ & $14.3 \pm 0.8$ \\
$193200.90 \pm 0.05^{*}$ & $+173144.4 \pm 0.8$ & $<1.2$ & $7.3 \pm 0.5$ & $7.0 \pm 0.8$ \\
$193059.85 \pm 0.04^{*}$ & $+173732.7 \pm 0.6$ & $<1.2$ & $9.0 \pm 0.5$ & $7.2 \pm 0.7$ \\
\hline
\end{tabular}

${ }^{a}$ Extended object. Apparent variability possibly affected by strong primary beam correction. 


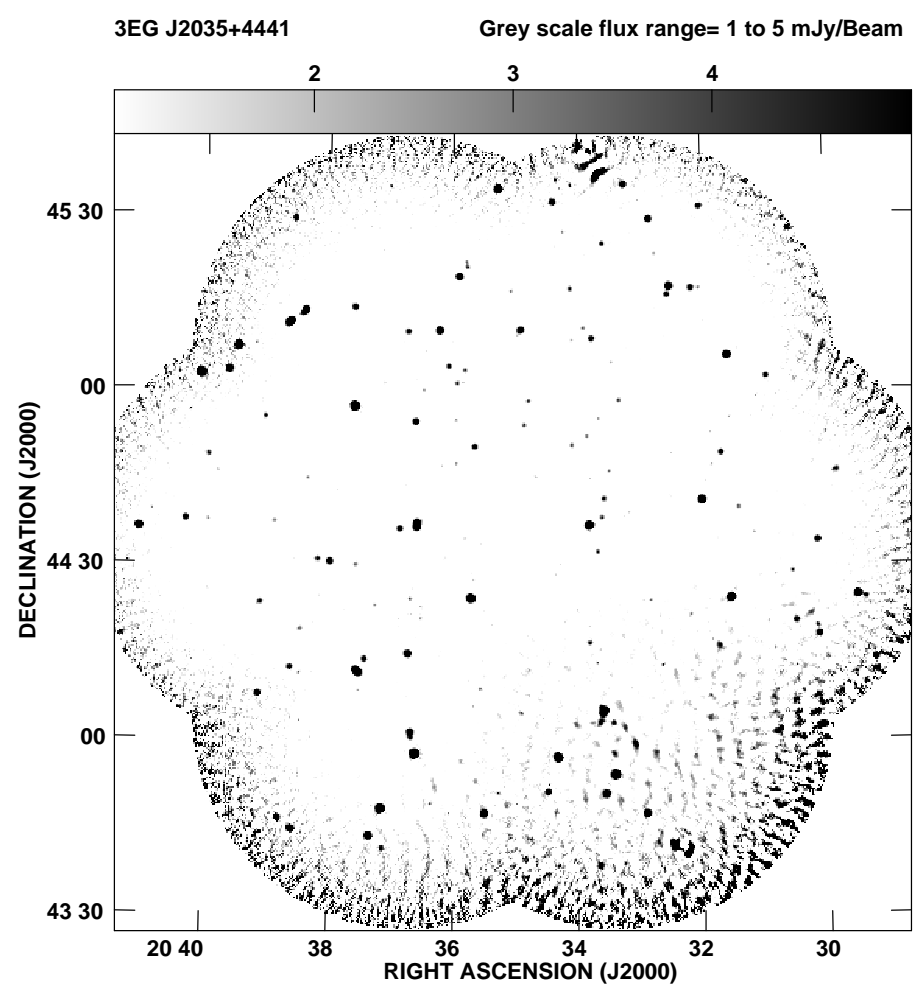

Figure 3. WSRT mosaic of the 3EG J2035+4441 field at $21 \mathrm{~cm}$ taken also on 2003 June 15 . Nearly 100 radio sources are detected in this image, but only a few of them display possible evidence of variability. A circular restoring beam of 45 " has been used as well.

Table III. Possible variable radio sources in the 3EG J2035+4441 field. The most reliable variables are marked with $\left(^{*}\right)$.

\begin{tabular}{ccccc}
\hline $\begin{array}{c}R A(2000) \\
(\mathrm{h} \mathrm{m} \mathrm{s})\end{array}$ & $\begin{array}{c}D E C(2000) \\
\left({ }^{\prime}, "\right)\end{array}$ & $\begin{array}{c}\text { NVSS } \\
(\mathrm{mJy})\end{array}$ & $\begin{array}{c}\text { WSRT1 } \\
(\mathrm{mJy})\end{array}$ & $\begin{array}{c}\text { WSRT2 } \\
(\mathrm{mJy})\end{array}$ \\
\hline $203731.42 \pm 0.01$ & $+441135.8 \pm 0.1$ & $91.6 \pm 3.3$ & $92.5 \pm 0.4$ & $87.5 \pm 0.7$ \\
$203634.34 \pm 0.01^{a}$ & $+443653.6 \pm 0.2$ & $80.6 \pm 3.0$ & $87.3 \pm 0.7$ & $79.1 \pm 0.7$ \\
$203604.26 \pm 0.05^{*}$ & $+450352.8 \pm 0.5$ & $<1.4$ & $7.2 \pm 0.3$ & $7.9 \pm 0.5$ \\
$203955.62 \pm 0.12$ & $+443738.9 \pm 1.2$ & $<1.4$ & $2.3 \pm 0.3$ & $2.0 \pm 0.4$ \\
$203057.85 \pm 0.03$ & $+450214.1 \pm 0.3$ & $7.1 \pm 0.6$ & $8.3 \pm 0.3$ & $9.0 \pm 0.5$ \\
$203702.85 \pm 0.25$ & $+445729.3 \pm 3.2$ & $<1.4$ & $<0.6$ & $2.2 \pm 0.5$ \\
\hline
\end{tabular}

${ }^{a}$ Double source. Position of brighter component given. 

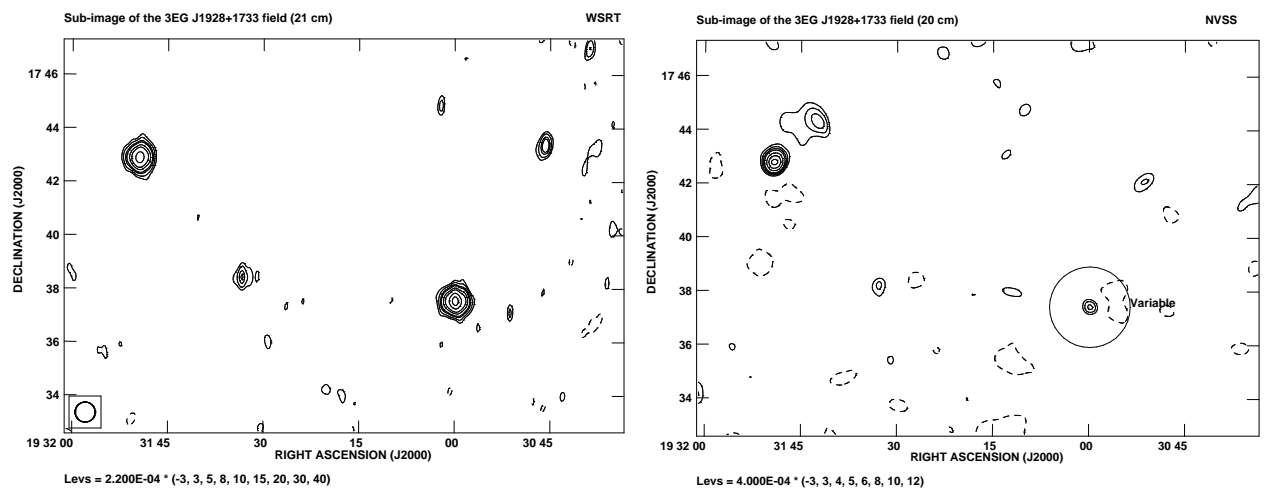

Figure 4. Example of a clearly compact variable radio source discovered when comparing our 3EG J1928+1733 mosaic (left panel) with the NVSS (right panel). The proposed variable source, indicated with a circle, appeared significantly fainter a few years before our WSRT observations.
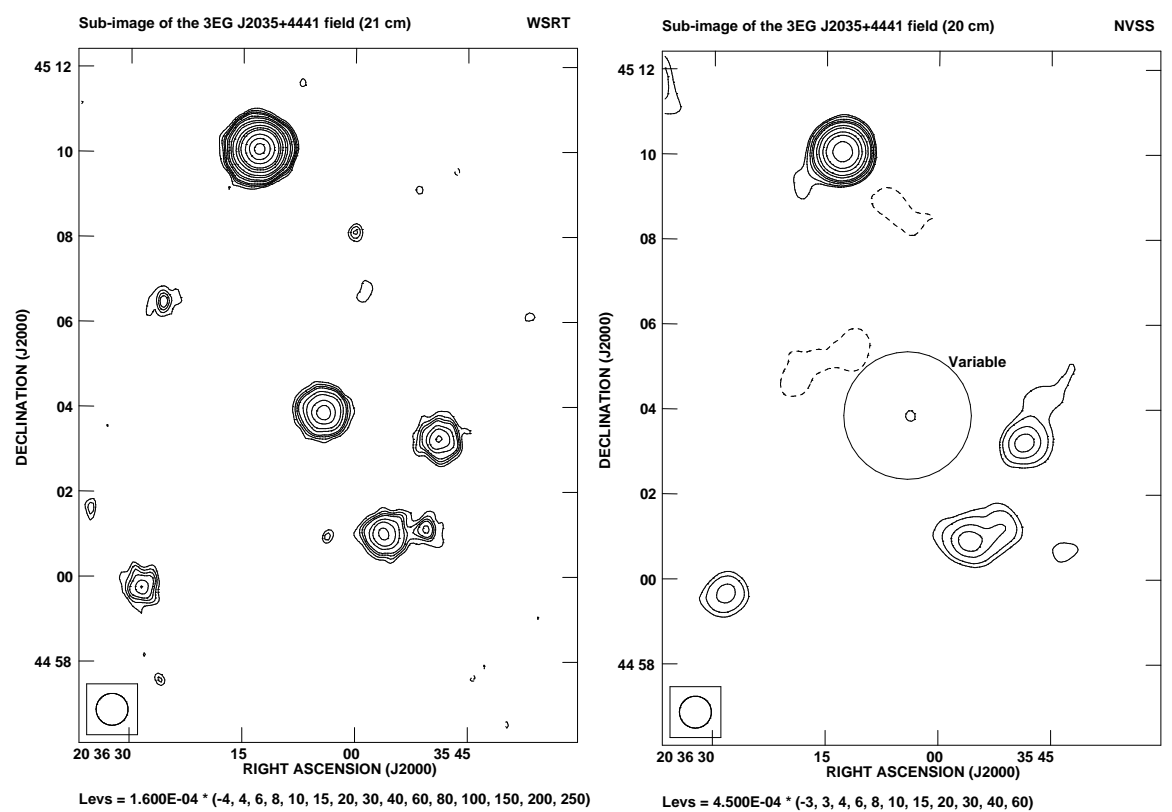

Figure 5. Example of a clearly variable and compact radio source discovered inside de $95 \%$ confidence contour of the unidentified EGRET source 3EG J2035+4441. The left panel is a zoomed region of a WSRT mosaic of the field as obtained by us on 2003 June 15 at $21 \mathrm{~cm}$. The right panel is the same region of the sky as it appeared in the NVSS in 1995. The proposed variable source, indicated with a circle, was practically undetectable some years ago. 
Table IV. Possible variable radio sources in the 3EG J1812-1316 field. The most reliable variables are marked with $\left({ }^{*}\right)$.

\begin{tabular}{cccc}
\hline $\begin{array}{c}R A(2000) \\
(\mathrm{h} \mathrm{m} \mathrm{s})\end{array}$ & $\begin{array}{c}D E C(2000) \\
\left({ }^{\circ}{ }^{\prime}\right)\end{array}$ & $\begin{array}{c}\text { NVSS } \\
(\mathrm{mJy})\end{array}$ & $\begin{array}{c}\text { VLA DDT } \\
(\mathrm{mJy})\end{array}$ \\
\hline $181352.65 \pm 0.13^{*}$ & $-133011.8 \pm 3.5$ & $6.4 \pm 0.5$ & $10.4 \pm 1.7$ \\
$181432.83 \pm 0.06$ & $-133037.4 \pm 1.8$ & $13.7 \pm 1.1$ & $18.9 \pm 1.6$ \\
$181426.88 \pm 0.06^{*}$ & $-132018.6 \pm 1.8$ & $12.0 \pm 0.6$ & $23.2 \pm 1.8$ \\
\hline
\end{tabular}

\section{Acknowledgements}

The Westerbork Synthesis Radio Telescope is operated by the ASTRON (Netherlands Foundation for Research in Astronomy) with support from the Netherlands Foundation for Scientific Research NWO. J.M.P., V.B-R. and J.M. acknowledge partial support by DGI of the Ministerio de Ciencia y Tecnología (Spain) under grant AYA-2001-3092, as well as additional support from the European Regional Development Fund (ERDF/FEDER). During this work, V.B-R. has been supported by the DGI of the Ministerio de Ciencia y Tecnología (Spain) under the fellowship FP-2001-2699. JM is also supported by the Plan Andaluz de Investigación (Spain) under project FQM322. The work of D.F.T. was performed under the auspices of the US DOE (NNSA), by UC's LLNL under contract No. W-7405-Eng-48. G.E.R. is supported by Fundación Antorchas and the Argentine Agencies CONICET and ANPCyT. This research has benefited from a cooperation agreement supported by Fundación Antorchas.

\section{References}

Bosch-Ramon V., Aharonian F.A., and Paredes J.M., 2004a, A\&A, submitted.

Bosch-Ramon V., Romero G.E., and Paredes J.M., 2004b, A\&A, submitted, [astro$\mathrm{ph} / 0405017]$

Bosch-Ramon V., Paredes, J.M., Romero G.E., and Torres, D.F., 2004c, in Proceedings of the Fifth Microquasar Workshop (Beijing).

Butt Y., Torres, D.F., Romero G.E. et al. 2002, Proc. of the 4th microquasar workshop, Cargese, France, May 2002. Ed. Ph. Durouchoux, Y. Fuchs \& J. Rodriguez. [astro-ph/0206132]

Combi J.A., Romero G.E., Paredes J.M., Torres D.F. and Ribó, 2003, ApJ 588, 731

Combi J.A., Romero G.E., Torres D.F., and Punsly B. 2001, ESA Special Publications, 459, 235 
Condon J.J., et al. 1998, AJ, 115, 1693

Dermer C.D., 1997, in Proceedings of the Fourth Compton Symposium, AIP, New York, p.1275.

Hapern et al., 2003, ApJ 125, 572

Hartman R.C., et al., 1999, ApJS 123, 79.

Kaufman-Bernadó M. M., Romero G. E., \& Mirabel I. F., 2002 A\&A 385, L10

Mattox J. R., Hartman R. C., Reimer O., 2001, ApJS 135, 155.

Paredes J.M., Martí J., Ribó M., \& Massi M. 2000, Sci, 288, 2340.

Punsly B., 1998, ApJ 498, 440.

Punsly B., Romero G.E., Torres D.F., Combi J.A., 2000, A\&A, 364, 552.

Ribó M., 2002, PhD Thesis, Universitat de Barcelona

Ribó M., García-Sánchez J., Paredes J.M., \& Martí J., 2004 A\&A (in preparation)

Romero G.E., Benaglia P., Torres D.F., 1999, A\&A 348, 868

Romero G.E., Kaufman-Bernadó M.M., Combi J., and Torres D.F., 2001 A\&A 376, 599.

Romero G. E., Kaufman-Bernadó M. M., \& Mirabel I. F., 2002 A\&A 393, L61

Sowards-Emmerd et al., 2003, ApJ, submitted (astro-ph/0212504)

Thompson D.J. 2001, AIP vol. 558, p. 103. Eds. F.A. Aharonian and H. J. Völk.

Torres D.F., Pessah M.E., and Romero G.E. 2001, Astron. Nach. 322, 223

Torres D.F., Romero G.E., Combi J.A., et al. 2001, A\&A, 370, 468.

Torres D.F., Romero G.E., Dame T.M., Combi J.A., and Butt Y.M. 2003, Physics Reports, Volume 382, Issue 6, p. 303

Address for Offprints: Josep M. Paredes

Departament d'Astronomia i Meteorologia

Facultat de Física. Universitat de Barcelona

Av. Diagonal 647

08028 Barcelona

Spain 